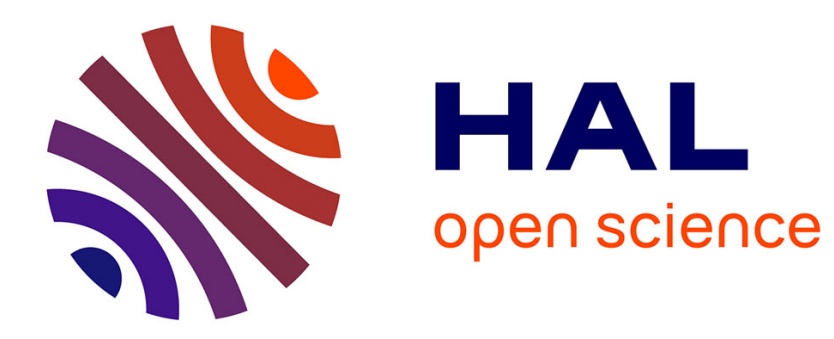

\title{
The variance of a rank estimator of transformation models
}

Koen Jochmans

\section{To cite this version:}

Koen Jochmans. The variance of a rank estimator of transformation models. 2011. hal-00973007

\section{HAL Id: hal-00973007 \\ https://hal-sciencespo.archives-ouvertes.fr/hal-00973007}

Preprint submitted on 21 May 2014

HAL is a multi-disciplinary open access archive for the deposit and dissemination of scientific research documents, whether they are published or not. The documents may come from teaching and research institutions in France or abroad, or from public or private research centers.
L'archive ouverte pluridisciplinaire HAL, est destinée au dépôt et à la diffusion de documents scientifiques de niveau recherche, publiés ou non, émanant des établissements d'enseignement et de recherche français ou étrangers, des laboratoires publics ou privés. 


\title{
THE VARIANCE OF A RANK ESTIMATOR OF TRANSFORMATION MODELS
}

\author{
Koen Jochmans* \\ CORE
}

First version: June 2010; This version: June 7, 2011

This note shows that the asymptotic variance of Chen's [Econometrica, 70, 4 (2002), 1683-1697] two-step estimator of the link function in a linear transformation model depends on the first-step estimator of the index coefficients.

JEL classification: C14, C41.

Keywords: influence function, transformation model, two-step estimator.

\section{The estimator}

For an unspecified strictly-increasing function $\Lambda_{0}(\cdot): \mathscr{R} \mapsto \mathscr{R}$, the linear transformation model takes the form

$$
\Lambda_{0}(Y)=X \beta+\varepsilon
$$

where $\varepsilon$ is a latent disturbance, distributed independently of the covariates $X$, and $\beta$ is an unknown coefficient vector of conformable dimension. Set $\Lambda_{0}\left(y_{0}\right)=0$ for a chosen baseline value $y_{0}$ and assume that $\beta=\left(1, \alpha_{0}^{\prime}\right)^{\prime}$.

Let $W_{i} \equiv\left(Y_{i}, X_{i}\right)(i=1, \ldots, n)$ be observations on $W \equiv(Y, X)$, drawn at random from a distribution $P$ that is supported on a set $\mathscr{W}$. Let $b_{n} \equiv\left(1, \alpha_{n}^{\prime}\right)^{\prime}$ be a first-step estimator of $\beta$. For fixed $y$, Chen (2002) proposed estimating $\Lambda_{0} \equiv \Lambda_{0}(y)$ by $\Lambda_{n}$, which maximizes

$$
\frac{1}{n(n-1)} \sum_{i \neq j} h\left(W_{i}, W_{j}, y, \Lambda, b_{n}\right), \quad h\left(W_{1}, W_{2}, y, \Lambda, b\right) \equiv\left[1\left\{Y_{1} \geq y\right\}-1\left\{Y_{2} \geq y_{0}\right\}\right] 1\left\{\left(X_{1}-X_{2}\right) b \geq \Lambda\right\}
$$

with respect to $\Lambda$ over a compact subset of the real line containing $\Lambda_{0}$.

\section{The influence function}

Impose Assumptions 1-5 of Chen (2002). Strenghten Assumption 6 by demanding $\alpha_{n}$ to be asymptotically linear, that is, $\sqrt{n}\left(\alpha_{n}-\alpha_{0}\right)=n^{-1 / 2} \sum_{i} \psi\left(W_{i}\right)+o_{p}(1)$ for a function $\psi(\cdot)$ that has zero mean and finite variance under $P$. Fix $y$ throughout and leave the dependence of quantities on it implicit. Let $\tau(w, \Lambda, b) \equiv$ $E h(W, w, y, \Lambda, b)+E h(w, W, y, \Lambda, b), V \equiv \frac{1}{2} E \nabla_{\Lambda \Lambda} \tau\left(W, \Lambda_{0}, \beta\right)$, and $\Omega \equiv E \nabla_{\Lambda \alpha^{\prime}} \tau\left(W, \Lambda_{0}, \beta\right)$. Arguments along the line of those in Sherman (1993) yield $\sqrt{n}\left(\Lambda_{n}-\Lambda_{0}\right)=n^{-1 / 2} \sum_{i} I\left(W_{i}\right)+o_{p}(1) \stackrel{\mathcal{L}}{\rightarrow} \mathcal{N}\left(0, E I(W)^{2}\right)$ for

$$
I(w) \equiv-V^{-1}\left[\nabla_{\Lambda} \tau\left(w, \Lambda_{0}, \beta\right)+\frac{1}{2} \Omega \psi(w)\right]=J(w)-\frac{1}{2} V^{-1} \Omega \psi(w) .
$$

Chen (2002, pp. 1687 and Theorem 1) argues that $\Omega=0$, so that $I(w)=J(w)$ and the asymptotic variance of $\sqrt{n} \Lambda_{n}$ is unaffected by the estimation noise in $b_{n}$.

*Address: Center for Operations Research and Econometrics, U.C. Louvain, Voie du Roman Pays 34, B-1348 Louvainla-Neuve, Belgium. Tel. +32 10474 329; E-mail: koen.jochmans@uclouvain.be. I am grateful to Songnian Chen, Geert Dhaene, Frank Kleibergen, and James Stock for comments and suggestions and to the Department of Economics of Brown University for their hospitality. 
Write $f$ and $p_{z}$ for the densities of $\varepsilon$ and $Z \equiv X \beta$, respectively. Arrange the components of $X=$ $\left(X_{1}, \widetilde{X}\right) \in \mathscr{R} \times \mathscr{X}$ so that the distribution of scalar $X_{1}$ given $\widetilde{X}=\widetilde{x}$ satisfies the absolute-continuity requirement of Chen (2002, Assumption 2) for all $\widetilde{x}$ in $\mathscr{X}$. The calculations summarized below show that, with $\mathcal{X}(z) \equiv E[\widetilde{X} \mid Z=z]$,

$$
\begin{aligned}
-V & =\int_{-\infty}^{+\infty} f(-z) p_{z}\left(z+\Lambda_{0}\right) p_{z}(z) \mathrm{d} z \\
\frac{1}{2} \Omega & =\int_{-\infty}^{+\infty} f(-z) p_{z}\left(z+\Lambda_{0}\right) p_{z}(z)\left[\mathcal{X}\left(z+\Lambda_{0}\right)-\mathcal{X}(z)\right] \mathrm{d} z .
\end{aligned}
$$

Equation (2) reveals that $\Omega$ will generally be non-zero. Like $V$, it can be estimated by the cross-derivative of a smoothed version of the symmetrized objective function evaluated at $\left(\Lambda_{n}, b_{n}\right)$. Consistency follows under conditions analogous to those for the estimator of $V$ stated in Chen (2002, pp. 1695-1696).

The conclusions drawn here extend to the case where the observations on $Y$ are subject to random censoring. The appropriate modification to the influence function stated in Chen (2002, Theorem 2) is readily derived.

\section{Calculations}

Let $\tau(w)=\tau(w, \Lambda, b)$ for fixed values $\Lambda$ and $b=\left(1, \alpha^{\prime}\right)^{\prime}$. Write $\tau_{0}(w)$ for $\tau\left(w, \Lambda_{0}, \beta\right), \nabla_{\Lambda} \tau_{0}(w)$ for $\nabla_{\Lambda} \tau\left(w, \Lambda_{0}, \beta\right)$, etc. Manipulate the inequalities in $\tau(w)$ to see that

$$
\begin{aligned}
\tau(W) & =\int_{\mathscr{W}}\left(1\left\{y<y_{0}\right\}-1\{Y<y\}\right) 1\{x b \leq X b-\Lambda\} \mathrm{d} P(w) \\
& -\int_{\mathscr{W}}\left(1\left\{Y<y_{0}\right\}-1\{y<y\}\right) 1\{x b<X b+\Lambda\} \mathrm{d} P(w)+c_{0}
\end{aligned}
$$

for $c_{0} \equiv \int\left(1\left\{Y<y_{0}\right\}-1\{y<y\}\right) \mathrm{d} P(y)$, which does not depend on $(\Lambda, b)$. Let $p_{z}(z \mid \widetilde{x})$ be the density of $Z$ given $\widetilde{X}=\widetilde{x}$ at $z$ and let $\Delta_{\alpha}(\widetilde{X}, \widetilde{x}) \equiv Z+(\widetilde{X}-\widetilde{x})\left(\alpha-\alpha_{0}\right)$. By iterated expectations,

$$
\tau(W)=-\int_{\mathscr{X}} \int_{-\infty}^{\Delta_{\alpha}(\widetilde{X}, \widetilde{x})-\Lambda} S_{y, y_{0}}(Y, z) p_{z}(z \mid \widetilde{x}) \mathrm{d} z \mathrm{~d} P(\widetilde{x})+\int_{\mathscr{X}} \int_{-\infty}^{\Delta_{\alpha}(\widetilde{X}, \widetilde{x})+\Lambda} S_{y_{0}, y}(Y, z) p_{z}(z \mid \widetilde{x}) \mathrm{d} z \mathrm{~d} P(\widetilde{x})+c_{0},
$$

where $S_{y_{1}, y_{2}}(Y, Z) \equiv 1\left\{Y<y_{1}\right\}-F\left(\Lambda_{0}\left(y_{2}\right)-Z\right)$ and $F(z) \equiv \int_{-\infty}^{z} f(z) \mathrm{d} z$.

Use Leibniz's rule to verify that

$$
\begin{aligned}
\nabla_{\Lambda} \tau(W) & =\int_{\mathscr{X}} S_{y, y_{0}}\left(Y, \Delta_{\alpha}(\widetilde{X}, \widetilde{x})-\Lambda\right) p_{z}\left(\Delta_{\alpha}(\widetilde{X}, \widetilde{x})-\Lambda \mid \widetilde{x}\right) \mathrm{d} P(\widetilde{x}) \\
& -\int_{\mathscr{X}} S_{y_{0}, y}\left(Y, \Delta_{\alpha}(\widetilde{X}, \widetilde{x})+\Lambda\right) p_{z}\left(\Delta_{\alpha}(\widetilde{X}, \widetilde{x})+\Lambda \mid \widetilde{x}\right) \mathrm{d} P(\widetilde{x})
\end{aligned}
$$

and that $\nabla_{\Lambda} \tau_{0}(W)=S_{y, y}(Y, Z) p_{z}(Z-\Lambda)-S_{y_{0}, y_{0}}(Y, Z) p_{z}(Z+\Lambda)$. Notice that $E \nabla_{\Lambda} \tau_{0}(W)=0$ because

$$
E\left[S_{y, y}(Y, Z) \mid Z=z\right]=0
$$

for any $y$. 
Differentiate with respect to $\Lambda$ under the integral sign in Equation (3), re-arrange, and evaluate at $\left(\Lambda_{0}, \beta\right)$ to obtain

$$
\nabla_{\Lambda \Lambda} \tau_{0}(W)=-f\left(\Lambda_{0}-Z\right) p_{z}\left(Z-\Lambda_{0}\right)-f(-Z) p_{z}\left(Z+\Lambda_{0}\right)-c_{1}
$$

where $c_{1} \equiv S_{y, y}(Y, Z) p_{z}^{\prime}\left(Z-\Lambda_{0}\right)+S_{y_{0}, y_{0}}(Y, Z) p_{z}^{\prime}\left(Z+\Lambda_{0}\right)$ and $p_{z}^{\prime}$ is the derivative of $p_{z}$. Integrate and apply the moment condition in Equation (4) to dispense with $c_{1}$ and to find that

$$
2 V=-\int_{-\infty}^{+\infty} f\left(\Lambda_{0}-z\right) p_{z}\left(z-\Lambda_{0}\right) p_{z}(z) \mathrm{d} z-\int_{-\infty}^{+\infty} f(-z) p_{z}\left(z+\Lambda_{0}\right) p_{z}(z) \mathrm{d} z
$$

Equation (1) follows on a change of variable from $z$ to $z-\Lambda_{0}$ in the first integral.

Follow the same steps to deduce that

$$
\nabla_{\Lambda \alpha^{\prime}} \tau_{0}(W)=f\left(\Lambda_{0}-Z\right) p_{z}\left(Z-\Lambda_{0}\right)\left[\widetilde{X}-\mathcal{X}\left(Z-\Lambda_{0}\right)\right]-f(-Z) p_{z}\left(Z+\Lambda_{0}\right)\left[\tilde{X}-\mathcal{X}\left(Z+\Lambda_{0}\right)\right]+c_{2}
$$

for $c_{2} \equiv S_{y, y}(Y, Z) p_{z}^{\prime}\left(Z-\Lambda_{0}\right)\left[\widetilde{X}-\mathcal{X}\left(Z-\Lambda_{0}\right)\right]-S_{y_{0}, y_{0}}(Y, Z) p_{z}^{\prime}\left(Z+\Lambda_{0}\right)\left[\widetilde{X}-\mathcal{X}\left(Z+\Lambda_{0}\right)\right]$. Because $c_{2}$ has zero mean,

$$
\Omega=\int_{-\infty}^{+\infty}\left\{f\left(\Lambda_{0}-z\right) p_{z}\left(z-\Lambda_{0}\right)\left[\mathcal{X}(z)-\mathcal{X}\left(z-\Lambda_{0}\right)\right]-f(-z) p_{z}\left(z+\Lambda_{0}\right)\left[\mathcal{X}(z)-\mathcal{X}\left(z+\Lambda_{0}\right)\right]\right\} p_{z}(z) \mathrm{d} z .
$$

A change of variable then establishes Equation (2).

\section{References}

Chen, S. (2002). Rank estimation of transformation models. Econometrica, 70:1683-1697.

Sherman, R. P. (1993). The limiting distribution of the maximum rank correlation estimator. Econometrica, 61:123-137. 